\title{
FACTORES DESCRIPTIVOS PARA LA \\ CONFORMACIÓN DE COOPERATIVAS \\ BINACIONALES: COSTA RICA-PANAMÁ
}

\author{
GUSTAVO HERNÁNDEZ CASTRO \\ Escuela de Ciencias de la Administración \\ Universidad Estatal a Distancia, Costa Rica \\ ghernandezc@uned.ac.cr \\ FEDERICO LI BONILLA \\ Escuela de Ciencias de la Administración \\ Universidad Estatal a Distancia, Costa Rica \\ fli@uned.ac.cr \\ ANGÉLICA CORDERO PRENDAS \\ Dirección de Extensión \\ Universidad Estatal a Distancia, Costa Rica \\ acorderop@uned.ac.cr
}

\section{RESUMEN}

El siguiente artículo explica los factores descriptivos determinantes que inciden en la conformación de un cooperativa binacional, para el caso de la región fronteriza entre Costa Rica y Panamá. La importancia de delimitar los factores descriptivos, radica en que estos, facilitarán comprender las expectativas de las personas y de las comunidades que habitan en la zona de estudio en el engranaje del desarrollo económico y social de esos espacios fronterizos.

Asimismo, se define las cooperativas binacionales como una forma de organización que tiene como propósito representar intereses económicos en territorios entre dos países, para el desarrollo económico y social de las naciones participantes, a su vez, implica normalizar aspectos jurídicos que le permitan funcionalidad y grado de independencia en su gestión administrativa.
PALABRAS CLAVE: COOPERATIVAS BINACIONALES, FACTORES DESCRIPTIVOS, COSTA RICA, PANAMÁ.

\section{ABSTRACT}

The following article explains the determinant descriptive factors affecting the establishment of binational cooperation in the border region between Costa Rica and Panama. The importance of defining these descriptive factors is that they will facilitate the understanding of the expectations of people and communities living in the area of study in relation to its economic and social development. Binational cooperation is defined as a form of organization that aims to represent economic interests between two countries, for economic and social development, and for normalizing legal aspects that allow functionality and a degree of independence.

KEYWORDS: BINATIONAL COOPERATIVE, DESCRIPTIVE FACTORS, COSTA RICA, PANAMÁ.

JEL Classification System: A13, J15, R58. 


\section{INTRODUCCIÓN}

El presente artículo presenta un avance de los resultados de la investigación titulada Los Factores descriptivos para la conformación de cooperativas binacionales: Costa Rica-Panamá.

Desde el (Plan Nacional de desarrollo "Jorge Manuel Dengo", 2005, p. 7) del gobierno de Costa Rica, se viene impulsando el Mapeo de la Cooperación Internacional en los cantones fronterizos de Costa Rica con Panamá; el mismo tiene como uno de sus propósitos "convertirse en insumos de primera mano para los actores locales, quienes tienen la responsabilidad de definir y llevar adelante sus procesos de desarrollo y definir sus estrategias de desarrollo localregional". Asimismo, estos mapeos obedecen a los convenios suscritos a nivel binacional entre ambos países y a los convenios reconocidos sobre "Cooperación para el Desarrollo Fronterizo, Ley Número 7518 del 10-07-1995, suscrito precisamente entre MIDEPLAN y el Ministerio de Economía y Finanzas (MEF) de Panamá, como instancias rectoras del desarrollo nacional de ambos países" (Plan Nacional de desarrollo "Jorge Manuel Dengo", 2005, p. 9).

De esta forma, y como insumo para apoyar los esfuerzos en el desarrollo comunitario de los cantones fronterizos, propuesto entre ambos países, se procedió a realizar una investigación con el propósito de conocer la percepción de los habitantes de esas zonas, para la conformación de cooperativas binacionales como ejes para el desarrollo local y sostenible de los cantones fronterizos y sus territorios. Para efectos de esta investigación, se encuestó residentes en los cantones de Coto Brus, Corredores provincia de Puntarenas, Costa Rica; y los distritos de Barú y Renacimiento; provincia de Chiriquí, Panamá.

\section{CONTEXTO}

Coto Brus es el cantón número 8 de la provincia de Puntarenas, Costa Rica. Su cabecera es San Vito; por su parte Corredores es el cantón número 10 de la provincia de Puntarenas; su cabecera es Ciudad Neily y es parte de la Región Brunca situada al sur de Costa Rica. Estos cantones son de los más ausentes en políticas públicas en lo que se refiere a Desarrollo Humano, vulnerabilidad infantil y pobreza.

Según la última Encuesta Nacional de Hogares realizada por el INEC (Julio de 2015) la Región Brunca, tiene $21,7 \%$ de pobreza no extrema y 13,7\% de pobreza extrema (ver tabla 1).

El cantón de Corredores se ubica en dirección noreste a suroeste, desde 300 metros al este de la naciente de quebrada Salitre, frontera con Panamá, hasta 1100 metros al sureste del origen del río La Vaca, límite con el citado país. Limita al norte con Coto Brus, al oeste con Golfito y al este y sur con Panamá. Se divide en 4 distritos: Corredor, La Cuesta, Canoas y Laurel.

El cantón de Coto Brus, limita al norte con el cantón de Buenos Aires; al este con la República de Panamá; al sur y oeste con Golfito y Buenos Aires. Coto Brus está a una hora de distancia de la zona turística panameña de Volcán-Bambito, provincia de Chiriquí y a dos horas de Golfito, punto de entrada al Parque Nacional Corcovado y a la Península de Osa, Costa Rica.

Chiriquí es una de las diez provincias de Panamá. Su capital es la ciudad de David. La provincia de Chiriquí se encuentra ubicada en el sector oeste de Panamá teniendo como límites al norte la provincia de Bocas del Toro y la comarca NgäbeBuglé, al oeste la provincia de Puntarenas (en la República de Costa Rica), al este la provincia de Veraguas y al sur el océano Pacífico.

La provincia de Chiriquí tiene $29,1 \%$ de pobres y $8,9 \%$ de indigentes. Sustantivo rescatar, que 
TABLA 1.

DISTRIBUCIÓN RELATIVA DE LOS HOGARES POR NIVEL DE POBREZA SEGÚN

REGIÓN DE PLANIFICACIÓN , JULIO 2014 Y 2015

\begin{tabular}{|c|c|c|c|c|c|c|c|c|c|c|}
\hline \multirow{2}{*}{$\begin{array}{l}\text { Zona y } \\
\text { región de } \\
\text { planifica- } \\
\text { ción }\end{array}$} & \multicolumn{5}{|c|}{2014} & \multicolumn{5}{|c|}{2015} \\
\hline & Total & $\begin{array}{c}\text { No } \\
\text { pobres }\end{array}$ & Total & $\begin{array}{c}\text { Pobreza } \\
\text { no } \\
\text { extrema }\end{array}$ & $\begin{array}{l}\text { Pobreza } \\
\text { extrema }\end{array}$ & Total & $\begin{array}{c}\text { No } \\
\text { pobres }\end{array}$ & Total & $\begin{array}{c}\text { Pobreza } \\
\text { no } \\
\text { extrema }\end{array}$ & $\begin{array}{l}\text { Pobreza } \\
\text { extrema }\end{array}$ \\
\hline Total & 100,0 & 77,6 & 22,4 & 15,7 & 6,7 & 100,0 & 78,3 & 21,7 & 14,6 & 7,2 \\
\hline \multicolumn{11}{|l|}{ Zona } \\
\hline Urbana & 100,0 & 80,5 & 19,5 & 14,2 & 5,2 & 100,0 & 80,6 & 19,4 & 13,7 & 5,7 \\
\hline Rural & 100,0 & 69,7 & 30,3 & 19,7 & 10,6 & 100,0 & 72,1 & 27,9 & 16,8 & 11,1 \\
\hline \multicolumn{11}{|c|}{ Región de planificación } \\
\hline Central & 100,0 & 82,8 & 17,2 & 12,8 & 4,5 & 100,0 & 82,9 & 17,1 & 12,4 & 4,7 \\
\hline Chorotega & 100,0 & 66,8 & 33,2 & 21,1 & 12,1 & 100,0 & 73,0 & 27,0 & 16,6 & 10,4 \\
\hline $\begin{array}{l}\text { Pacífico } \\
\text { Central }\end{array}$ & 100,0 & 70,5 & 29,5 & 19,7 & 9,7 & 100,0 & 72,6 & 27,4 & 17,3 & 10,1 \\
\hline Brunca & 100,0 & 63,8 & 36,2 & 24,7 & 11,6 & 100,0 & 64,6 & 35,4 & 21,7 & 13,7 \\
\hline Huetar Caribe & 100,0 & 71,8 & 28,2 & 19,9 & 8,3 & 100,0 & 71,0 & 29,0 & 17,9 & 11,1 \\
\hline Huetar Norte & 100,0 & 73,2 & 26,8 & 17,3 & 9,6 & 100,0 & 72,8 & 27,2 & 16,7 & 10,5 \\
\hline
\end{tabular}

Nota: en porcentajes. Por INEC, Encuesta Nacional de Hogares (ENAHO) 2014, 2015.

FIGURA 1.

UBICACIÓN DEL CANTÓN DE CORREDORES Y COTO BRUS EN EL MAPA DE COSTA RICA

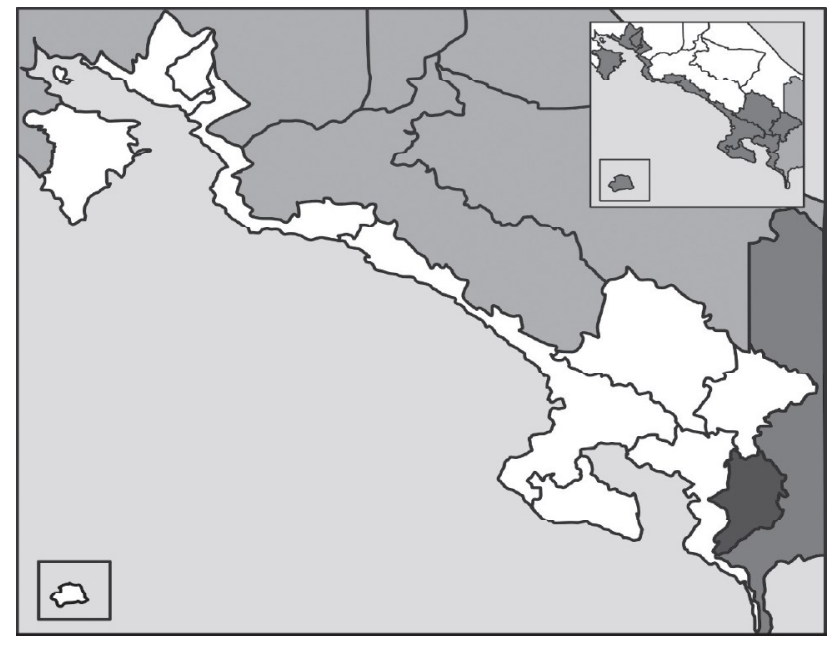

Fuente: Google Maps (2015). 
según el INEC de Panamá, citado por Diéguez y Alvarado (2012), en cuanto a la categoría ocupacional, las cooperativas contribuyen a solucionar los paliativos de la pobreza, debido a que estas contribuyen en $81,9 \%$ a que las población estén en condiciones de bienestar social, es decir personas no pobres, 17,7\% familias que están en pobrezas extrema y o, 5 en pobreza extrema, estas últimas cifras, relacionadas con emprendimientos productivos no focalizados o adecuadamente gestionados.

El distrito de Barú tiene como cabecera el Puerto Armuelles. Se fundó en 1941; tiene una extensión de 595 km2 y una población 55800 habitantes (censo 2010). Su principal actividad económica es el cultivo del banano y la producción de aceite de palma. Se divide en los corregimientos de: Puerto Armuelles, Limones, Progreso, Baco y Rodolfo Aguilar Delgado.

Por su parte Renacimiento tiene como cabecera Río Sereno. Se fundó en 1971; tiene una extensión de 529 km2 y una población 20600 habitantes (censo 2010). Al igual que Barú, su principal actividad económica es el cultivo del banano y la producción de aceite de palma. Se divide en los siguientes corregimientos de: Breñón, Cañas Gordas, Dominical, Monte Lirio, Plaza Caisán, Río Sereno, Santa Cruz y Santa Clara.

Según (Diéguez y Alvarado, 2012, p. 10) la pobreza general y la extrema son las más comunes en el área rural ya que "(...) está ligada a la calidad del empleo, que es precaria en las empresas familiares rurales, así como en los trabajos por cuenta propia, poco remunerados en las zonas rurales si se le compara, para la misma ocupación, al de la urbana".

En este contexto, una forma de coadyuvar a la reducción de la pobreza (Región Brunca; cantón de Corredores y de Coto Brus, Costa Rica, y los cantones de Barú y Renacimiento, Chiriquí, Panamá) es el establecimiento de cooperativas binacionales, por cuanto, esta modalidad permitiría aprovechar las capacidades sociales y económicas de ambos zonas fronterizas y en consecuencia la movilidad social de sus habitantes.

Por su parte, el Ministerio de Planificación y Política Económica del gobierno de Costa Rica en su Plan Nacional de Desarrollo [PND] (2014) establece:

FIGURA 2

\section{UBICACIÓN DEL DISTRITO BARÚ EN EL MAPA DE PANAMÁ}

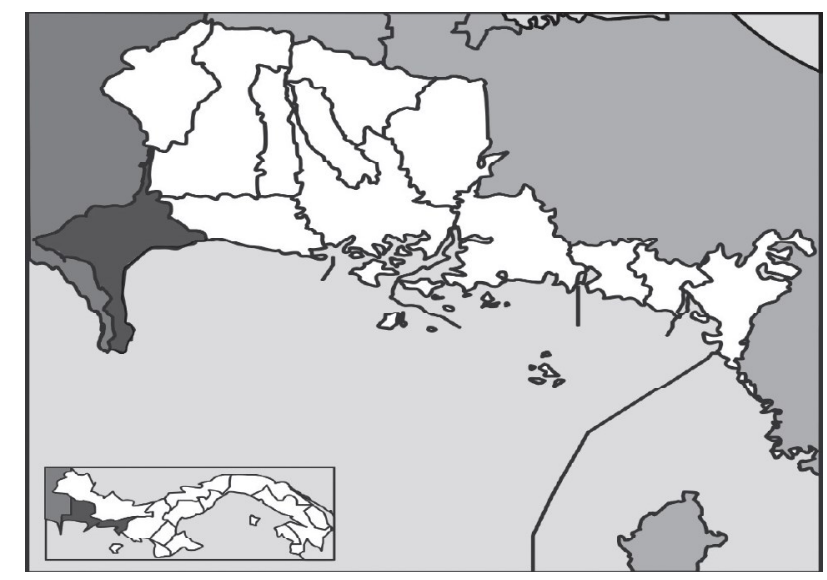

Fuente: Google Maps (2015).. 


\section{UBICACIÓN DEL DISTRITO RENACIMIENTO EN EL MAPA DE PANAMÁ}

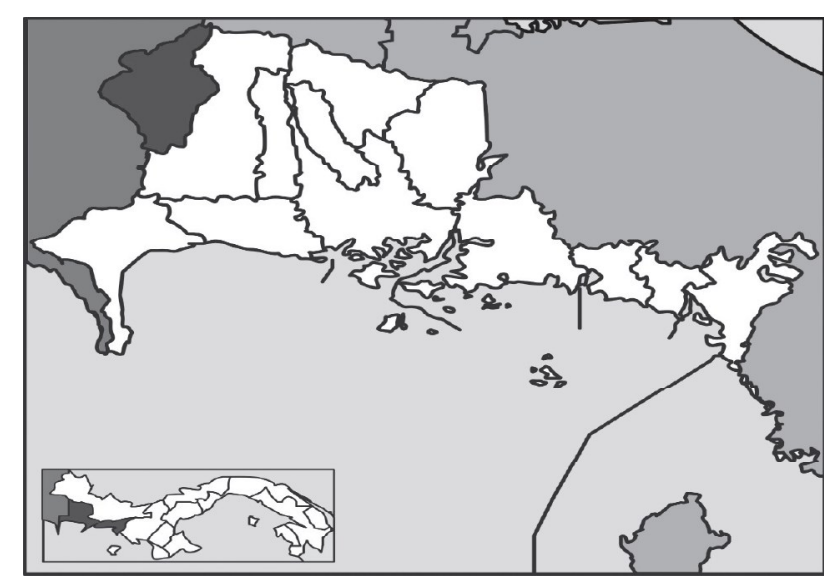

Fuente: Google Maps (2015).

Apoyar la meta nacional de reducción de la pobreza mediante acciones que mejoren las condicio $\neg$ nes de vida en los territorios rurales y propicien la dignificación de la población rural. Se plantea unan reducción de la pobreza rural en 6.5 puntos porcentuales; siempre que sea una meta conjunta entre varios sectores, especialmente de los sectores: Trabajo y Seguridad Social; Desarrollo Humano e Inclusión Social; Educativo; Salud, Nutrición y Deporte; Vivienda y Asentamientos Humanos, entre otros. Por otro lado es necesario que se den las condiciones macroeconómicas adecuadas para que el aporte del sector sea efectivo en el ámbito nacional (p. 292).

Asimismo, el punto 1.4.4.1 denominado Micros, pequeñas y medianas agroempresas comercializando o en el mercado institucional, establece como meta para el periodo 2015-2018 la conformación de 20 MiPymes en la Región Brunca (PND, 2014, p. 299). Aprovechando el PND (2014) del país (Estado) y mediante los esfuerzos que se puedan realizar en conjunto con los gobiernos locales, organizaciones sociales, movimiento cooperativo, entre otras instituciones, potenciarían una solución sustantiva para elevar los índices de desarrollo de las comunidades anfitriones en las zonas geográficas ya mencionadas, las cooperativas binacionales, sería un solución para elevar los índices de desarrollo y potenciar las capacidades económicas y sociales de las fronteras entre Costa Rica y Panamá.

\section{Las empresas binacionales}

La conformación y creación de las empresas binacionales tiene relación con acuerdos bilaterales entre dos naciones o Estados. Se pude afirmar que es una figura empresarial hibrida entre sociedades anónimas, públicas y cooperativas (y similares) y la participación de los Estados, en la cual estos últimos, deben garantizar el marco jurídico (bilateral) para que la relaciones económicas entre las partes se lleven a cabo.

White (1987) señala que las empresas binacionales son concebidas como empresas de propiedad conjunta de inversionistas nacionales de dos países para el fortalecimiento del capital nacional (ambos países). 
Las empresas binacionales operan en un espacio concreto (las fronteras), así pues, existe una correlación directa entre actividades económicas y el tema de los espacios fronterizos. No se debe perder de vista que las organizaciones binacionales actúan en estos espacios geográficos. Estas vienen a reconocer la idiosincrasia de un territorio divido por una frontera política.

De esta forma, Otálvora (2003) dedica un análisis exhaustivo sobre este tema e indica que la política exterior de un Estado, es parte de la lógica propia de las relaciones generales entre las naciones colindantes y adquieren características resultantes de la diversidad de instancias institucionales (o no-institucionales) y áreas temáticas que dan cuerpo a las relaciones binacionales. En este caso en particular, existe la etnia indígena común a los países lo que presiona la homogenización de ideales y necesidades en común propias de un territorio.

Por su parte Maris y Raya de Vera (2007) establecen que el factor determinante para la constitución de una empresa binacional es el trato preferencial de propiedad conjunta y supone que por lo menos $80 \%$ del capital social y de los votos pertenezca a inversionistas nacionales; que se asegure a estos el control real y efectivo de la empresa y que por lo menos la participación de inversores de cada uno de los dos países tenga derecho a elegir, como mínimo, un miembro de los órganos de administración y de fiscalización interna de la empresa.

Para el caso de Paraguay, ABC (2008) señala que las divisas que ingresan a la economía producto de las organizaciones binacionales, no se encuentran normadas, ya que no forman parte de las estimaciones del PIB, a diferencia de Brasil, Argentina, inclusive Uruguay, que son registradas como parte del indicador macroeconómico.

Etcheverry (2008) define a las empresas binacionales como entes públicos (organizaciones intergubernamentales), con forma asociativa empresaria, que tiene un estatuto de característica autorregulatoria y deben ser considerados "nacionales" en cada uno de los Estados partes.

Cracogna, D. (2009) se refiere al acuerdo macro de la creación del Estatuto de las Cooperativas del Mercosur en la cual se justifica en que las cooperativas de un Estado puedan brindar sus servicios a personas domiciliadas en otro u otros Estados, no solo en actividades económicas, sino también incorporándolos como asociados.

García (2014) refiriéndose al caso de Argentina y Brasil, señala que una empresa binancional es aquella que cumpla (simultáneamente) con un capital, no menos del 80\%, y el control efectivo deben estar en manos de inversiones nacionales. La participación efectiva de los inversores nacionales de cada país no debe ser inferior al 30\% del capital social.

Al analizar los referentes conceptuales previos se puede definir las cooperativas binacionales como una forma de organización que tiene como finalidad representar intereses económicos entre dos países, para el desarrollo económico y social entre las naciones participantes, a su vez, implica normalizar aspectos jurídicos que le permitan funcionalidad y grado de independencia en su gestión administrativa.

\section{METODOLOGÍA}

Esta investigación exploratoria, transversal de corte mixta; comprende investigación de campo representado por encuestas (34 variables observables); además la triangulación realizada conlleva la revisión de políticas de estado (PND) y las diferentes consultas que los pobladores de la zona hacían a la extensionista participante de esta investigación sobre posibilidades reales de fuentes de trabajo compartidas entre ambas franjas fronterizas. Adicionalmente se realizó una revisión exhaustiva de lo escrito sobre cooperativas binacionales. 


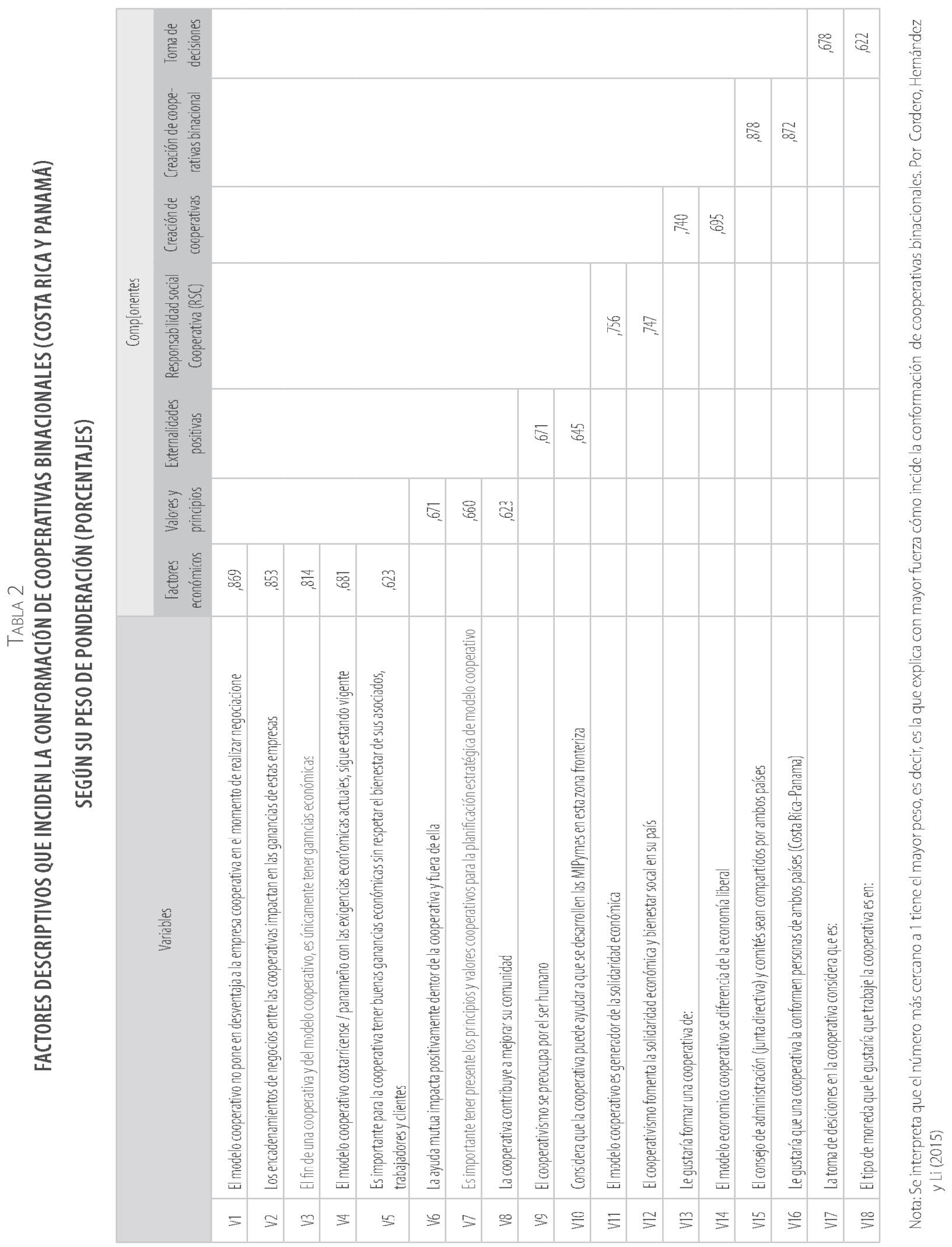


Las consultas realizadas comprendieron a estudiantes universitarios de ambas fronteras, así como estudiantes de último año de colegios técnicos de secundaria; y pequeños comerciantes y agricultores de las zonas limítrofes.

Se utilizó un instrumento que contiene 34 variables (preguntas cerradas) basadas en la escala de Liker (No sé; no respondo, en total descuerdo, en desacuerdo, de acuerdo y totalmente de acuerdo), Schwarzer y Jerusalem (1995). En la tabla 2 se presenta un resumen de 18 principales variables con la que se trabajó para la redacción de este artículo.

El levantamiento de los datos se recolectaron entre los meses de febrero 2014 a setiembre de 2015 en las zonas fronterizas ya indicadas para un total de 400 encuestas (200 por país) y una vez depuradas por el SSPS19 quedaron válidas 392 encuestas.

\section{Población de estudio}

Se encuestaron 392 personas distribuidos, de estas 197 son personas que habitan la parte de Costa Rica y 195 el lado de Panamá; con una confianza del $95 \%$ y un error máximo permitido de $3 \%$, es decir, toda diferencia superior al 3\%, entre las variables observadas, es evidencia de diferencia significativa.

De esta población encuestada (tabla 3) 208 corresponden a mujeres y 178 hombres.

Adicionalmente, se tomó como parámetro (al momento de aplicar la encuestas) a aquellas personas que tuvieran conocimiento limitado sobre el cooperativismo o bien que no trabajaran en cooperativas, lo anterior, para reducir un sesgo a favor o en contra de la organización cooperativa.

TABLA 3

POBLACIÓN ENCUESTADA

\begin{tabular}{|c|c|c|c|c|c|}
\hline & & ecuencia & Porcentaje & Porcentaje válido & $\begin{array}{l}\text { Porcentaje } \\
\text { acumulado }\end{array}$ \\
\hline \multirow{3}{*}{ Válidos } & Costa Rica & 197 & 50,3 & 50,3 & 50,3 \\
\hline & Panamá & 195 & 49,7 & 49,7 & 100,0 \\
\hline & Total & 392 & 100,0 & 100,0 & \\
\hline
\end{tabular}

Nota: En frecuencias y porcentajes. Por Cordero, Hernández y Li (2015).

TABLA 4

GÉNERO DE LOS ENTREVISTADOS

\begin{tabular}{|c|c|c|c|c|c|}
\multicolumn{2}{|c}{} & \multicolumn{2}{c}{ Frecuencia } & \multicolumn{2}{c|}{$\begin{array}{c}\text { Porcentaje } \\
\text { Porcentaje } \\
\text { válido }\end{array}$} \\
\hline \multirow{3}{*}{ Válidos } & Masculino & 178 & 45,4 & 46,1 & 46,1 \\
\cline { 2 - 6 } & Femenino & 208 & 53,1 & 53,9 & 100,0 \\
\cline { 2 - 6 } & Total & 386 & 98,5 & 100,0 & \\
\cline { 2 - 6 } & Perdidos sistema & 6 & 1,5 & & \\
\hline \multirow{2}{*}{ Total } & Total & 392 & 100,0 & & \\
\hline
\end{tabular}

Nota: En frecuencias y porcentajes. Por Cordero, Hernández y Li (2015). 
TABLA 5

CONOCE CÓMO FUNCIONA UNA COOPERATIVA

\begin{tabular}{|c|c|c|c|c|c|}
\hline & & Frecuencia & Porcentaje & $\begin{array}{l}\text { Porcentaje } \\
\text { válido }\end{array}$ & $\begin{array}{l}\text { Porcentaje } \\
\text { acumulado }\end{array}$ \\
\hline \multirow{4}{*}{ Válidos } & incorrecto & 78 & 19,9 & 19,9 & 19,9 \\
\hline & Apenas cierto & 109 & 27,8 & 27,8 & 47,7 \\
\hline & Más bien cierto & 110 & 28,1 & 28,1 & 75,8 \\
\hline & Cierto & 95 & 24,2 & 24,2 & 100,0 \\
\hline Total & Total & 392 & 100,0 & 100,0 & \\
\hline
\end{tabular}

Nota: En frecuencias y porcentajes. Por Cordero, Hernández y Li (2015).

\section{Análisis de variables}

Se procedió al análisis de las variables con el propósito e identificar las subyacentes, o bien, que explicaran la configuración de correlaciones dentro de las 34 variables observadas. De estas, 18 explican por qué (modelo) se conformar la cooperativa de capital y asociados binacional (de Costa Rica y Panamá: sector fronterizo) empresa cooperativa inédita a nivel global. A su vez, estas variables se agruparon en siete componentes a saber:

- Factores económicos

- Valores y principios cooperativos

- Externalidades cooperativas positivas

- Responsabilidad social cooperativa

- Creación de cooperativas

- Toma de decisiones cooperativas

Es importante resaltar el orden de importancia que se da con el análisis multivariado de factores. Según Li (2013) este método que escruta el factor que manifieste la cantidad principal y de mayor significancia sobre la varianza en la matriz. De cada factor se obtiene una valoración propia asociada, que corresponde a una varianza. Esta varianza es una comunalidad, es decir, una cuantía de varianza de la pregunta analizada con las demás preguntas sometidas a investigación.

Con este análisis factorial sin rotar, la matriz obtenida brinda las contribuciones de las preguntas o variables que dan una fuerte correlación al componente o constructo, es decir, la vinculación entre las preguntas y el componente o factor obtenido. Una característica muy importante cuando se realiza un análisis factorial sin rotar, es la alta relación de variables dentro del componente propuesto.

El análisis de los componentes principales es la transformación lineal en un sistema diferente de coordenadas al agregado de información para la cual la varianza de mayor importancia se da en el primer pilar, conocido como el principal componente o constructo, siendo sus variables las originales e independientes entre sí; el cual explica en un determinado porcentaje el modelo propuesto.

El análisis factorial o reducción de factores, se obtiene por ciento de la varianza total. De tal manera que el porcentaje obtenido brinda un valor el cual determina si es el análisis es válido o no. Se consideran válidos los resultados si los factores explican al menos 95\% en las ciencias naturales; para el caso de esta investigación y de las ciencias sociales es frecuente un resultado del 60 al $65 \%$ del total de la varianza. 
En cuanto a la interpretación de los resultados en cada factor, se debe tener en cuenta que el mínimo requerido es de $\pm 0,30$, de forma tal que valores de $\pm 0,40$ son importantes a considerar y valores superiores a $\pm 0,50$ son significativos. Para el análisis de esta investigación se consideró, como parámetro mínimo para la reducción de factores y tal como se indicó en el análisis de reducción de factores, el límite igual o superior a 0,50 de los diferentes factores que arrojara el modelo multifactorial. Es así como los resultados obtenidos superiores a 0,50 en su valor absoluto, se consideran variables de mucho peso e importantes en la determinación de la muestra.

\section{Hallazgos de la investigación}

De las 34 variables analizadas, 18 de ellas, revisten un carácter sustantivo que se correlaciona con los siete componentes o factores descriptivos como condición sine qua non para la conformación de las cooperativas binacionales en el espacio geográfico (fronteras) determinado (tabla 4). Estas obtuvieron un peso superior al 0,50.

En torno al componente Factor económico (variable 1 a la 5):

V1: El modelo cooperativo no pone en desventaja a la empresa cooperativa en el momento de realizar negociaciones. Un temor válido (pero no justificante) es que el las empresas instaladas y las personas que habitan en el espacio fronterizo no visualizan a las cooperativas como un elemento competitivo que vaya a restar o eliminar de mercado a estas empresas; es decir, la conformación de cooperativas bajo el modelo binacional cuenta con el respaldo comunitario.

V2. Los encadenamientos de negocios entre las cooperativas impactan en las ganancias de estas empresas. Al conformarse cooperativas entre ambas fronteras, estas provocarían encadenamientos productivos y de comercialización que dinamizaría la actividad económica, es decir empleo, al haber mayor población ocupada, habrá un flujo de capital y en consecuencia poder adquisitivo de compra que impactarían las ganancias de las cooperativas.

V3: El fin de una cooperativa y del modelo cooperativo, es tener únicamente ganancias económicas. Se desprende de esta variable, que las cooperativas, son un factor de movilidad social (bienestar para sus asociados) en tanto que empresa, debe liderarse bajo los principios de eficiencia y productividad.

V4: El modelo cooperativo Costarricense/ Panameño con las exigencias económicas actuales, sigue estando vigente. Las cooperativas son un motor de desarrollo y un vínculo para que los asociados y las comunidades anfitrionas (Hernandez, 2013), sean promotoras del desarrono local y social.

La V5: Es importante para la cooperativa el tener buenas ganancias económicas sin respetar el bienestar de sus asociados, trabajadores y clientes. Variable relaciona con la V14; 59,4\% de los encuestados considera que es incorrecto que una cooperativa no respete el bienestar de sus asociados, y 15,3\% lo consideren apenas cierto. En otras palabras las ganancias económicas son importantes y necesarias, como es inherente el respecto por el bienestar del asociado, trabajadores y clientes.

En cuanto al componente Valores y principios cooperativos (variable 6 a la 8):

V6: La ayuda mutua impacta positivamente dentro de la cooperativa y fuera de ella. Se desprende que la organización cooperativa tiene una gran significación social y solidaria puesto que la percepción es su impacto positivo entre las personas.

V7: Es importante tener presente los principios y valores cooperativos para la planificación estratégica del modelo cooperativo. Tema que tiene 
relación directa con la gerencia estratégica. Una cooperativa exitosa es una organización que se rige bajo los criterios de la ciencia administrativa y por tanto los principios y valores cooperativos deben enmarcarse desde la gestión estratégica.

V8. La cooperativa contribuye a mejorar su comunidad. Variable directa con el componente de Factores económicos; es indiscutible, la percepción existente de ambos lados fronterizos en que la organización cooperativa es un motor de desarrollo comunitario.

Respecto al componente Externalidades cooperativas (variable 9 y 10 ):

V9. El cooperativismo se preocupa por el ser humano. Ligada al componente de Valores y principios, el cooperativismo en su esencia, está ligado al bienestar individual y social del ser humano. La práctica empresarial cooperativa debe empoderar al colectivo en las metas de productividad y prosperidad mediante la dignificación del trabajo y salarios competitivos según la necesidad y capacidad de cada asociado.

V10: Considera que la cooperativa pueda ayudar a que se desarrollen las MiPymes en esta zona fronteriza. Relacionada con la V2 (Factores económicos), las cooperativas no solo son un puente para el desarrollo de las MiPymes, sino también, estas se puedan desempeñar con un criterio de MiPymes, al desarrollar encadenamientos de negocios y productivos para las comunidades anfitrionas.

En relación con el componente Responsabilidad Social Cooperativa (variable 11 y 12):

V11: El modelo cooperativo es generador de la solidaridad económica. Vinculadas con las V8 y 9 , se desprende que las cooperativas sustentan participaciones solidarias tanto de sus asociados como dentro del radio de acción de su actividad cconómica (comunidades anfitrionas) por tal motivo, la conformación de cooperativas entre ambas fronteras potenciaría las capacidades sociales en procura de un mayor bienestar económico y social.

V12: El cooperativismo fomenta la solidaridad económica y bienestar social en su país. Si la organización cooperativa es promotora del desarrollo social en sus comunidades anfitrionas, en consecuencia, estas contribuirían al fortalecimiento de Producto Interno Bruto (PIB).

Vinculado al componente Creación de cooperativas (variable 13 y 14):

V13: Le gustaría conformar una cooperativa de. Se desprende del estudio que la mayoría de los encuestados si quieren conforman un cooperativa ya sea Tradicional; Cogestión o Autogestión. El modelo de cooperativa tradicional tuvo el mayor aceptación ya que los asociados a este tipo de organización, no tiene la obligación de trabajar en ella, como así es en lo otros modelos (cogestión y autogestión). Importante destacar que la conformación de cooperativas en ahorro y crédito, servicios, producción, turismo, servicios múltiples, energía, educación y agroindustria son el tipo de cooperativas que llaman la atención conformaren los espacios fronterizos.

V14: El modelo económico cooperativo se diferencia de la economía liberal. Conexo a la variable V5 se instituye que las cooperativas por su esencia velan por la seguridad y bienestar del asociado-trabajador, es decir, se aleja de la economía del mercado representadas por compañías, en la cual los accionistas son los que se benefician de las utilidades y del giro propio del negocio, en tanto, para satisfacer las demandas del mercado, contratan personal, contra una remuneración. En las cooperativas los asociados son los propios "accionistas" y por tanto, velan por el bienestar de todos.

Afín al componente Creación de cooperativas binacionales (variable 15 y 16): 
V15: El consejo de administración (junta directiva) y comités sean compartidos por ambos países. Es un tema que tiene relación directa con la gerencia estratégica y la normativa jurídica existente entre países. No obstante, como señala Hernandez, (2014) el Manual de Balanza de Pagos y Posición de Inversión Internacional del FMl, emite criterios vinculantes para los países que clasifican una persona como residente para trabajar en un país diferente del suyo.

V16: Le gustaría que una cooperativa la conformaran personas de ambos países (Costa Rica-Panamá)

Relacionado al componente Toma de decisiones (variable 17 y 18):

V17: La toma de decisiones en la cooperativa considera que es. Los estilos de liderazgo que se establezcan, producto de una gestión estratégica es la base por la cual las cooperativas puedan ser exitosas. La toma de decisiones es vinculante con un estilo de liderazgo asertivo; para 33,9\% la toma decisiones en una cooperativa es muy compleja; y 26,5\% la consideran compleja. Se desprende que los tomadores de decisiones deberían ser personas con competencias probadas en la gestión administrativa cooperativa.

V18: El tipo de moneda que le gustaría que trabaje la cooperativa es en. Los resultados obtenidos arrojaron que 43,4\% respondió operar sus emprendimientos cooperativos con el dólar americano para sus distintas transacciones y 50,8\% la utilización de ambos monedas (dólares americanos y colones costarricenses).

Un hallazgo encontrado a partir de un análisis transversal, a luz de esta investigación, y que puede coadyuvar esfuerzos para aliviar la situación de los trabajadores transfronterizos, es la normativa establecida en el Manual de Balanza de Pagos y Posición de Inversión Internacional del Fondo Monetario Internacional (FMI), que emite criterios vinculantes para los países y que clasifican a una persona como residente para trabajar en un país diferente del suyo. La movilidad de trabajadores transfronterizos para realizar trabajos agrícolas y de comercio en época estacionales (temporada) merece de atención ya que la remuneración reciba por estos y las condiciona laborales en las cuales trabajan no les permite superar y elevar su nivel de vida; es un Tal y como lo señala (Hernández, 2014, p. 39) "La realidad es que los trabajadores migrantes y transfronterizos se ganan \$273 dólares mensuales en la cosecha del melón (\$9,10 por día) [...] \$193,73 mensuales en la cosecha de café (\$6,46 por día) [...] y $\$ 336,37$ dólares mensuales en el comercio fronterizo (\$11,21 por día)". Esfuerzos cooperativos en los espacios fronterizos señalados, aliviarían aún más la problemática de los trabajadores transfronterizos y de temporada, en tanto que el modelo cooperativo, es generador de solidaridad económica.

\section{CONCLUSIONES}

Se definen las cooperativas binacionales como una forma de organización que tiene como finalidad representar intereses económicos entre dos países, para el desarrollo económico y social entre las naciones participantes, en este caso, existe una idiosincrasia, basada en rasgos culturales, de idioma autóctono, sustentado en el libre tránsito histórico en una franja de 30 kilómetros a cada lado de la frontera; a su vez, implica normalizar aspectos jurídicos que le permitan funcionalidad y grado de independencia en su gestión administrativa.

Se realizó una triangulación mediante la revisión de políticas de estado (PND) y las diferentes consultas que los pobladores de la zona realizaban a la extensionista participante de esta investigación sobre las posibilidades reales de fuentes de trabajo compartidas entre ambo espacios fronterizos. 
Se procedió al análisis de las variables con el propósito e identificar las subyacentes, o bien, que explicaran la configuración de correlaciones dentro de las 34 variables observadas. De estas, 18 explican por qué se debe conformar la cooperativa de capital y asociados binacional (de Costa Rica y Panamá: sector fronterizo).

Un factor determinante para la conformación de cooperativas binacionales, radica en la posibilidad, de aprovechar las capacidades económicas y sociales de ambos lados fronterizos para que los emprendimientos cooperativos a establecer lleguen a ser exitosos en el tiempo.

Estos emprendimientos productivos, deben ir enfocados en la conformación de cooperativa de tipo Tradicional, ya que los asociados a este tipo de organización, no tiene la obligación de trabajar en ella, como así es en lo otros modelos (cogestión y autogestión).

En el marco de la cooperativa "tradicional" se subraya la conformación de cooperativas con los siguientes giros empresariales: ahorro y crédito, servicios, producción, turismo, servicios múltiples, energía, educación y agroindustria son el tipo de cooperativas que llaman la atención conformaren los espacios fronterizos.

Menester señalar que entre ambos espacios fronterizos, los valores y principios, las externalidades, la Responsabilidad Social Cooperativa (RSC), entre otros componentes del estudio, son determinantes positivos para la conformación de los emprendimientos cooperativos, en otras palabras, el terreno está arado y abonado para la siembra de nuevos proyectos cooperativos, que culminarían exitosamente entre ambos países (Costa Rica y Panamá).

Otro tema de atención en la conformación de cooperativas binacionales (Costa Rica y Panamá) que implicaría realizar una investigación de carácter longitudinal es la relación y los resultados a largo plazo del Mapeo de la Cooperación
Internacional en los cantones fronterizos de Costa Rica con Panamá (Ministerio de Planificación Nacional y Política Económica, 2005) y la propuesta de Corredor Mesoamericano de Integración. Integrando Mesoamérica por el Pacífico, liderado por el Banco Interamericano de Desarrollo (Infante, 2012). Ya que ambos proyectos Tienen la finalidad de impactar positivamente las actividades económicas de las zonas fronterizas y de la región centroamericana con Mesoamérica a partir de la integración física y comercial

Finalmente, uno de los resultados de esta investigación, resalta la coincidencia del punto VI: "Sectores de desarrollo nacional apoyados por la cooperación en los cantones fronterizos con Panamá" del Mapeo de la Cooperación Internacional en los cantones fronterizos de Costa Rica con Panamá (Ministerio de Planificación Nacional y Política Económica, 2005, p. 50), en la cual describe los siguiente sectores como ejes de proyectos de ejecución de las zonas: productivo; ambiente; energía y telecomunicaciones; lucha contra la pobreza; coordinación gubernamental; turismo y salud; y cultura. Estos sectores coindicen con la opinión de los encuestados en que las cooperativas binacionales realicen emprendimientos en esos sectores de interés.

Como corolario, se da por aceptado la creación de una cooperativa binacional, en el territorio estudiado, y la necesidad de esta, para paliar, los bajos ingresos de los habitantes, a su vez sea una fuente de desarrollo del territorio transfronterizo, costarricense panameño.

\section{REFERENCIAS}

ABC. (2008). Binacionales, fuera del PIB y su discusión está en agenda. Recuperado de: http://www.abc. com.py/edicion-mpresa/economia/binacionales-fuera-del-pib-y-su-discusion-esta-en-agenda-1093412.html. (5 de abril de 2015). 
Cracogna, D. (2009). El Estatuto de las Cooperativas del Mercosur. Boletín de la Asociación Internacional de Derecho Cooperativo, 17-32.

Diéguez, J., Alvarado, R. (2012). Indigencia y pobreza. Encuesta de mercado de trabajo. Ministerio de Economía y Finanzas, Panamá.

Etcheverry, R. (2014). Empresas binacionales argentinobrasileras: un nuevo instrumento de integración. Recuperado de: http://www.juridicas.unam.mx/ publica/librev/rev/revdpriv/cont/8/dtr/dtr3.pdf (5 de setiembre de 2014).

García, J. (2014). Empresas binacionales argentinobrasileñas. Recuperado de: http://www.abeledogottheil.com.ar/uploads/c2725fc2fe4bf884b49c4e851400bfb5917e69b5.pdf (10 de marzo 2015).

Hernández, G. (2013). Gestión de calidad en las empresas turísticas sostenibles. Costa Rica: EUNED.

Hernández, G. (2014). Reflexiones sobre los trabajadores transfronterizos y de temporada: análisis del Informe metodológico del Banco Central de Costa Rica: trabajadores transfronterizos y de temporada en Costa Rica 2011-2012. Revista Nacional de Administración. 5 (2), 27-40,.

INEC (2014). Encuesta Nacional de Hogares. Costa Rica: INEC.

Infante, I. (2012). Corredor Mesoamericano de Integración. Integrando Mesoamérica por el Pacífico. Revista Instituto para la Integración de América Latina y el Caribe, 34, año 16, Enero-Junio.
Li, F. (2013). Modelo cooperativo costarricense. Costa Rica: EUNED.

Ministerio de Planificación Nacional y Política Económica. (2005). Plan Nacional de Desarrollo (20062010) "Jorge Manuel Dengo". Costa Rica: MIDEPLAN.

Ministerio de Planificación Nacional y Política Económica. (2014). Plan Nacional de Desarrollo (20152018) "Alberto Cañas Escalante". Costa Rica: MIDEPLAN.

Otálvora, E. (2003). Frontera en tiempos de globalización. El Proyecto ZIF. Revista Venezolana de Economía y Ciencias Sociales, 9(1), 85-114.

White, E. (1987). Empresas binacionales argentinobrasileras: una propuesta para su promoción. En Revista Integración Latinoamericana. Recuperado de : http://www10.iadb.org/intal/intalcdi/ integracion_latinoamericana/documentos/129Estudios_3.pdf (5 de setiembre de 2014).

Schwarzer; R., Jerusalem, M. (1995). Generalized SelfEfficacy scale. In J. Weinman, S. Wright, \& M. Johnston (Eds), Measures in health psicologic: A user portafolio. Casual and control beliefs (pp. 35-37). Windosr. UK: NEFER-NELSON

Recibido: 10 de octubre de 2016 Aceptado: 30 de marzo de 2016 
This document was created with Win2PDF available at http://www.win2pdf.com. The unregistered version of Win2PDF is for evaluation or non-commercial use only. This page will not be added after purchasing Win2PDF. 